\title{
Prédigestion d'un composant du lait, le lactose, dans la glande mammaire
}

Depuis l'avènement de la transgénèse dans les années 1980 , de nombreux projets ont eu pour objectif d'appliquer cette technologie à l'amélioration de l'alimentation humaine. Si elle est couramment utilisée pour les plantes, la transgénèse reste marginale chez l'animal. Beaucoup d'études ont cherché à modifier le lait, aussi bien dans le cadre de la production de médicaments que dans celui de l'alimentation humaine. En effet, la glande mammaire constitue un bioréacteur très performant, la récolte du lait est aisée, et les études concernant la spécificité d'expression des gènes de la glande mammaire ont permis de caractériser des promoteurs utilisables en transgénèse.

Le lait est un aliment riche en macro et micronutriments. Le lactose est le sucre majeur du lait $(5 \mathrm{~g} / 100 \mathrm{ml}$ de lait de vache) et joue le rôle de pompe osmotique dans la glande mammaire pour fluidifier le lait. Pour être digéré, le lactose doit être hydrolysé en glucose et en galactose par la lactase, $\beta$-galactosidase digestive présente exclusivement dans l'intestin grêle chez les mammifères. L'activité lactasique est importante à la naissance, à l'exception des cas rares d'alactasie congénitale. Elle se maintient à un niveau élevé chez $30 \%$ des individus adultes (d'origine caucasienne et de certaines ethnies d'Afrique et d'Asie), mais, chez $70 \%$ d'entre eux, chute vers l'âge de 25 ans et atteint chez l'adulte une valeur 10 à 20 fois plus faible que celle du nourrisson $[1,2]$. Le déclin d'activité lactasique définit le phénotype d'hypolactasie et entraîne une maldigestion du lactose. Chez les individus conservant une activité lactasique élevée à l'âge adulte, une hypolactasie transitoire peut se déclarer lors de maladies digestives. Le lactose du lait ingéré par les individus hypolacta- un déséquilibre osmotique dans l'intestin grêle. Dans le côlon, le lactose est métabolisé en acides gras volatils et en gaz par la flore bactérienne colique, expliquant les symptômes cliniques qui résultent de la maldigestion du lactose et qui associent des douleurs abdominales et une diarrhée osmotique pouvant conduire à une déshydratation sévère $[2,3]$.

La richesse nutritionnelle du lait en fait un aliment de choix, y compris pour les adultes. Dans les pays industrialisés, la consommation de produits laitiers couvre $10 \%$ des apports énergétiques, $75 \%$ des besoins en calcium (intérêt dans la prévention de l'ostéoporose), et $20 \%$ à $40 \%$ des besoins en riboflavine, phosphore, magnésium et vitamine B12 [4]. Le lait est également utilisé comme complément nutritionnel dans les programmes d'aide alimentaire destinés aux pays en voie de développement. Or l'hypolactasie limite considérablement la consommation de lait à l'âge adulte et représente de ce fait un problème de santé à l'échelle planétaire. Cela a justifié le développement de méthodes permettant de réduire la teneur en lactose. Elles sont de deux types : d'une part, l'utilisation de préparations orales (liquides ou tablettes) de $\beta$-galactosidase de levure consommées en même temps que le lait; d'autre part, le traitement industriel du lait qui élimine le lactose par l'action d'une $\beta$-galactosidase ou par ultracentrifugation. Toutefois, si ces produits sont bien tolérés par les individus hypolactasiques, leur distribution reste limitée en raison de leur coût généralement élevé.

Les contraintes liées au traitement industriel du lait ont conduit à rechercher des méthodes alternatives de production in vivo de lait appauvri en lactose, en utilisant la technologie de la transgénèse chez la souris. Une façon d'y parvenir consiste à empê- cher la synthèse de lactose dans les cellules mammaires en invalidant le gène de la sous-unité régulatrice de la lactose synthétase: l' $\alpha$-lactalbumine [5]. Cependant, l'absence totale de lactose rend le lait extrêmement visqueux et des recherches sont actuellement en cours pour tenter de limiter, sans empêcher totalement, la production d' $\alpha$-lactalbumine, ce qui assurerait une synthèse réduite de lactose et normaliserait ainsi l'osmolarité du lait. La stratégie employée est celle des ribozymes [6], mais son efficacité est difficile à contrôler lors de la transmission du caractère dans la descendance des animaux transgéniques.

L'objectif de la seconde approche est de diminuer la teneur en lactose du lait en le «prédigérant» dans la glande mammaire. Le principe consiste à construire des animaux transgéniques qui sécrètent dans le lait l'enzyme lactase capable d'hydrolyser le lactose. Celle-ci est synthétisée sous la forme d'un précurseur enzymatiquement actif [7]. Pour tester la faisabilité de cette méthode, nous avons placé la synthèse de lactase sous le contrôle d'un promoteur spécifique de la glande mammaire : le promoteur de l' $\alpha$-lactalbumine [8]. Chez les souris transgéniques allaitantes, le précurseur de la lactase est effectivement synthétisé puis exporté dans le lait à la surface des globules lipidiques. L'activité lactasique présente dans le lait provoque une diminution spécifique de la teneur en lactose de $50 \%$ à $85 \%$, sans altérer sa viscosité ni sa composition en protéines et en graisses. Le glucose et le galactose libérés lors de l'hydrolyse du lactose sont largement réabsorbés par les cellules mammaires. Le lait modifié conserve ses propriétés nutritionnelles et son absorption n'entraîne aucun effet secondaire. Ces résultats valident la méthode employée et suggèrent qu'elle pourrait être appliquée aux bovins qui 
produiraient alors in vivo un lait appauvri en lactose. En effet, une réduction de $50 \%$ à $70 \%$ de la teneur en lactose est suffisante pour éliminer les symptômes cliniques que crée la maldigestion du lactose [9]. Ce travail constitue un des premiers succès dans l'utilisation de la méthode de la transgénèse chez l'animal à des fins d'alimentation humaine. Des tentatives précédentes avaient échoué en raison de la trop faible expression du caractère génétique recherché ou en raison d'effets secondaires indésirables [10]. Le succès de ce travail est lié au fait que la lactase, même si elle est faiblement exprimée dans le lait, est suffisamment active pour réduire la teneur en lactose sans toutefois provoquer les inconvénients liés à l'absence totale de ce disaccharide.

Néanmoins, il est difficile de prévoir quelle sera l'exploitation future de cette approche car plusieurs problèmes restent en suspens. On connaît mal l'accueil qui serait fait par les consommateurs au lait provenant d'OGM (ce point dépasse le cadre de cet article). Par ailleurs, l'application de la méthode décrite aux bovins soulève des questions spécifiques : (1) l'hydrolyse du lactose sera-t-elle aussi efficace dans le lait de vache (contenant $5 \mathrm{~g} / 100 \mathrm{ml}$ de lactose) qu'elle ne l'est dans celui de la souris (2 g/ $100 \mathrm{ml}$ ) ? (2) Faudra-t-il augmenter le niveau d'expression de la lactase dans la glande mammaire ? (3) Le goût du lait est-il modifié, puisque le lactose est réduit et que le glucose et le galactose sont en partie réabsorbés par les cellules mammaires? Plusieurs années de recherche seront nécessaires pour répondre à ces questions.

1. Simoons FJ. Geographic patterns of primary adult lactose malabsorption: a further interpretation of evidence for the Old World. In : Paige DM, Bayless TM, eds. Lactose digestion: clinical and nutritional implications. Baltimore: John Hopkins University Press, 1981 : 23-48.

2. Sahi T. Genetics and epidemiology of adulttype hypolactasia. Scand J Gastroenterol $1994 ; 29$ (suppl 202) : 7-20.

3. Suarez FL, Savaiano DA, Levitt MD. The treat- ment of lactose intolerance. Aliment Pharmacol Ther $1995 ; 9: 589-97$.

4. National Dairy Council. Nutritional implications of lactose and lactase activity. Dairy Counc Dig $1985 ; 56$ : 25-30.

5. Stinnakre MG, Vilotte JL, Soulier S, Mercier JC. Creation and phenotypic analysis of $\alpha$-lactalbumin-deficient mice. Proc Natl Acad Sci USA 1994 ; $91: 6544-8$.

6. L'Huillier PJ, Soulier S, Stinnakre MG, et al. Efficient and specific ribozyme-mediated reduction of bovine $\alpha$-lactalbumin expression in double transgenic mice. Proc Natl Acad Sci USA 1996 ; 93 : 6698-703.

7. Jost B, Duluc I, Richardson M, Lathe R, Freund JN. Functional diversity and interactions between the repeat domains of rat intestinal lactase. Biochem J 1997 ; 327 : 95-103.

8. Jost B, Vilotte JL, Duluc I, Rodeau JL, Freund JN. Production of low-lactose milk by ectopic expression of intestinal lactase in the mouse mammary gland. Nat Biotech $1999 ; 17: 160-4$.

9. Vesa TH, Korpela RA, Sahi T. Tolerance to small amounts of lactose in lactose maldigesters. Am J Clin Nutr 1996 ; 64 : 197-201.

10. Whitelaw B. Toward designer milk. Nat Biotech $1999 ; 17: 135-6$.

\section{Jean-Noël Freund}

Inserm U. 381, 3, avenue Molière, 67200 Strasbourg, France.

\section{DRÈVES}

\begin{abstract}
-1 Hypothyroïdie au cours d'un essai clinique d'un ligand spécifique du récepteur RXR. La mise au point de nouveaux ligands pour les récepteurs nucléaires de la famille des récepteurs de l'acide rétinoïque est l'un des domaines les plus actifs de le recherche pharmacologique. Ces travaux ont été encouragés par l'efficacité de l'acide rétinoïque dans le traitement de certaines leucémies et en dermatologie. Les rétinoïdes ont deux familles principales de récepteurs, les RAR et les RXR. Ces derniers reconnaissent l'acide 9-cis rétinoïque, mais sont aussi capables de former des hétérodimères actifs avec un grand nombre de récepteurs nucléaires (voir $\mathrm{m} / \mathrm{s}$ 1998, $n^{\circ} 11$, p. 1211 et $1999, n^{\circ} 6 / 7$, p. 885-7). Plusieurs molécules liant préférentiellement les isoformes du RXR sont actuellement en développement. Une étude récente décrit certaines observations d'un essai clinique utilisant l'une de ces molécules, le bexa-
\end{abstract}

rotène, par voie orale, chez des patients souffrant de lymphome à cellules $\mathrm{T}$ de localisation cutanée [1]. Les doses ont été ajustées pour éviter l'apparition de leucopénie et d'hypertriglycéridémie. Chez 26 patients sur 27 étudiés, la concentration sérique de TSH a baissé sous traitement pour atteindre des valeurs nettement inférieures aux valeurs normales. Il en est de même de la thyroxinémie et de la concentration de triiodothyronine. Cette diminution était accompagnée chez plusieurs patients de manifestations cliniques d'hypothyroïdie, en particulier d'une intolérance au froid, qui ont nécessité un traitement par la thyroxine. L'ensemble de ces symptômes cliniques et biologiques a régressé à l'arrêt du traitement. Une expérience sur cellules en culture a permis de confirmer qu'un agoniste du récepteur RXR inhibait l'activité du promoteur du gène de la sousunité TSH $\beta$. Cette dernière observa- tion confirme un travail précédent montrant que les agonistes du récepteur RXR et la triiodothyronine réprimaient le promoteur du gène de la TSH $\beta$ de manière additive et agissaient par l'intermédiaire de séquences promotrices différentes [2]. Il est donc probable que ces ligands du RXR n'agissent pas par l'intermédiaire de l'hétérodimère TR-RXR, ce qui est d'ailleurs en accord avec l'observation clinique d'hypothyroïdie. Ces observations permettent de mieux connaître les possibilités et les limites de l'utilisation de ces molécules. Il est intéressant, mais sans doute un peu facile après coup, de remarquer que les effets cliniques étaient prévisibles au vu des données expérimentales précédentes.

[1. Sherman SI, et al. $N$ Engl J Med 1999; 340: 1075-9.]

[2. Haugen BR, et al. Mol Endocrinol 1997; 11: 481-9.] 